\title{
High-power single-mode fiber amplifiers using multimode fibers
}

\author{
M.E. Fermann, A. Galvanauskas, D. Harter, J.D. Minelly, * J.E. Caplen \\ * IMRA America, 1044 Woodridge, Avenue, Ann Arbor, Michigan 48105 \\ -Optoelectronics Research Center, Southampton, S017/BJ, UK
}

As the optical power levels extractable from current single-mode (SM) fiber amplifiers are reaching regions where optical nonlinearities become significant, even under $\mathrm{cw}$ operation, ${ }^{1}$ it is becoming increasingly important to develop methods for reducing these nonlinearities to further boost the amplifier performance.

Here we demonstrate a simple new approach, namely the SM excitation of specially designed large-core multimode (MM) fibers. Cladding-pumped $\mathrm{Er} / \mathrm{Yb}$ amplifier versions of such fibers allow the direct amplification of diffraction-limited optical soliton pulses with peak powers up to $12 \mathrm{~kW}$, about one order of magnitude larger than possible with conventional fiber amplifiers.

Under SM excitation of a MM mode fiber, the amount of power propagating in the fundamental mode as a function of fiber length decreases due to micro-bending-induced mode-coupling. The resulting $\mathrm{M}^{2}$-value as a function of fiber length, as calculated from mode-coupling experiments, in various $50-\mu \mathrm{m}$-core diameter fibers is shown in Fig. 1. The excitation wavelength was $1.55 \mu \mathrm{m}$. In this fiber \#1 was made by the rod-in-tube technique, whereas fibers $\# 2$ and $\neq 3$ where made using modified chemical-vapor deposition. The low-micro-bending fiber $\# 3$ indeed allows to obtain an $\mathrm{M}^{2}$ value $<1.2$ for fiber lengths up to $10 \mathrm{~m}$, allowing for essentially diffractionlimited amplification in cladding-pumped $\mathrm{Er} / \mathrm{Yb}$ amplifiers, which typically vary in length between $1-10 \mathrm{~m}$.

The fundamental mode is launched in these MM fibers with high accuracy by suppressing modal speckle by using broad-bandwidth excitation sources such as ultrashort pulses. As the temporal coherence length of broad-bandwidth sources is very short, temporal interference between the modes and thus the presence of speckle is prevented. The stability of the spatial beam profile was verified by coupling the output of a MM fiber into a SM dummy fiber and measuring the launched signal power as a function of time. The result for both single-frequency (SF)

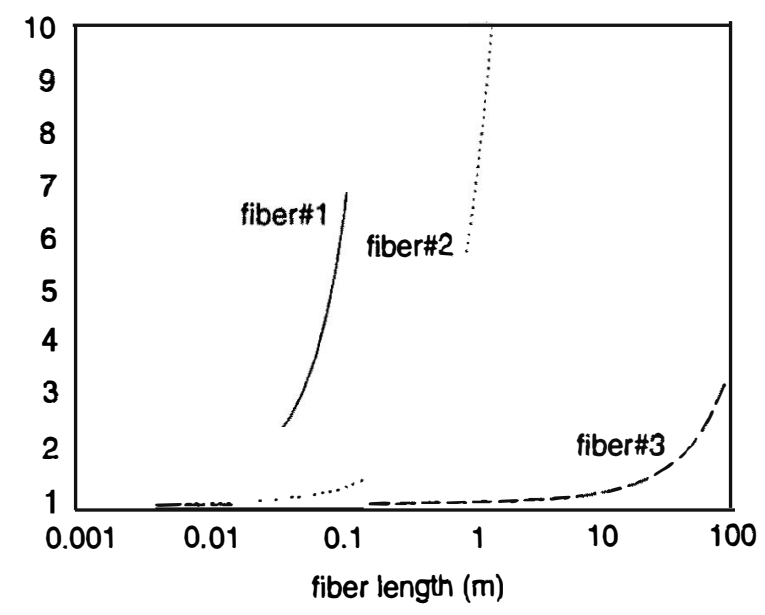

Fig. 1. $\mathrm{M}^{2}$-values at a wavelength of $1.55 \mu \mathrm{m}$ in $50-\mu \mathrm{m}$-core diameter fibers of different design as a function of fiber length.

excitation and broad-band excitation of the MM fiber is shown in Fig. 2. The large random power fluctuations due to modal speckle for the case of SF excitation are clearly visible, whereas the power fluctuations for broad-band excitation are about a factor of 5 smaller.
Figure 3 shows the autocorrelation and the spectrum of $340 \mathrm{fs}$ pulses directly amplified to a peak power of $12 \mathrm{~kW}$ in a $1.5 \mathrm{~m}$ length of 30 - $\mu \mathrm{m}$-core diameter cladding-pumped $\mathrm{Er} / \mathrm{Yb}$ amplifier fiber. The pulses had an energy of $4 \mathrm{~nJ}$ and were near-transform-limited with a time-bandwidth-product of 0.29 . The autocorrelation is displayed on a logarithmic scale to demonstrate the absence of a pedestal. Secondary autocorrelation peaks due to small residual excitation of higher-order modes are suppressed to better than $0.5 \%$.

Pulses amplified to similar peak powers in such $\mathrm{Er} / \mathrm{Yb}$ fibers have recently also been frequency-doubled to generate $300 \mathrm{fs}$ femtosecond pulses with peak powers of $25 \mathrm{~kW}$ and a record average power of $300 \mathrm{~mW}$ at $780 \mathrm{~nm}$.

In conclusion, we have demonstrated a new technique for greatly expanding the operation limits of fiber lasers. The very large-core, low micro-bending fiber amplifiers discussed here should allow the construction of a new generation of ultrahigh-power fiber laser systems.

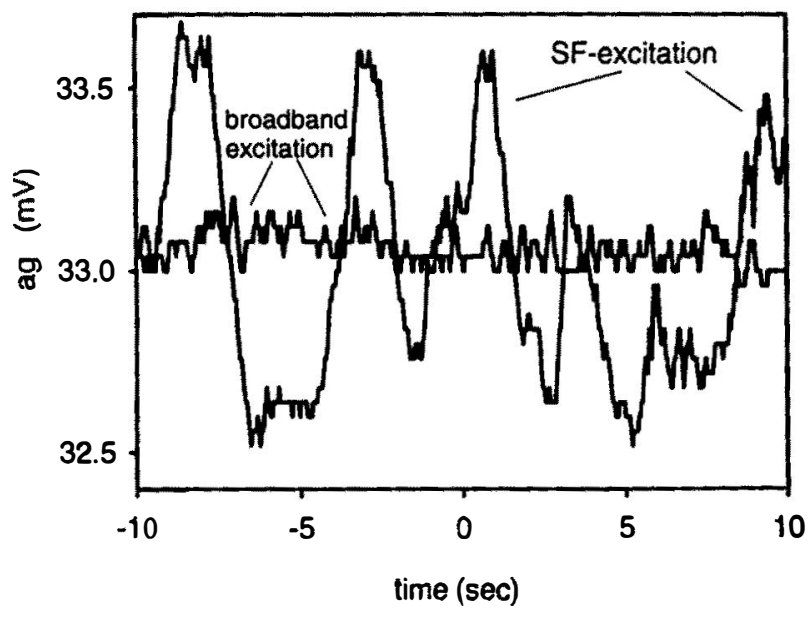

Fig. 2. Noise level of the output from a $30-\mu \mathrm{m}$-core diameter MM fiber when coupled into a SM dummy fiber under both spectral broad-band and single-frequency (SF) excitation. 

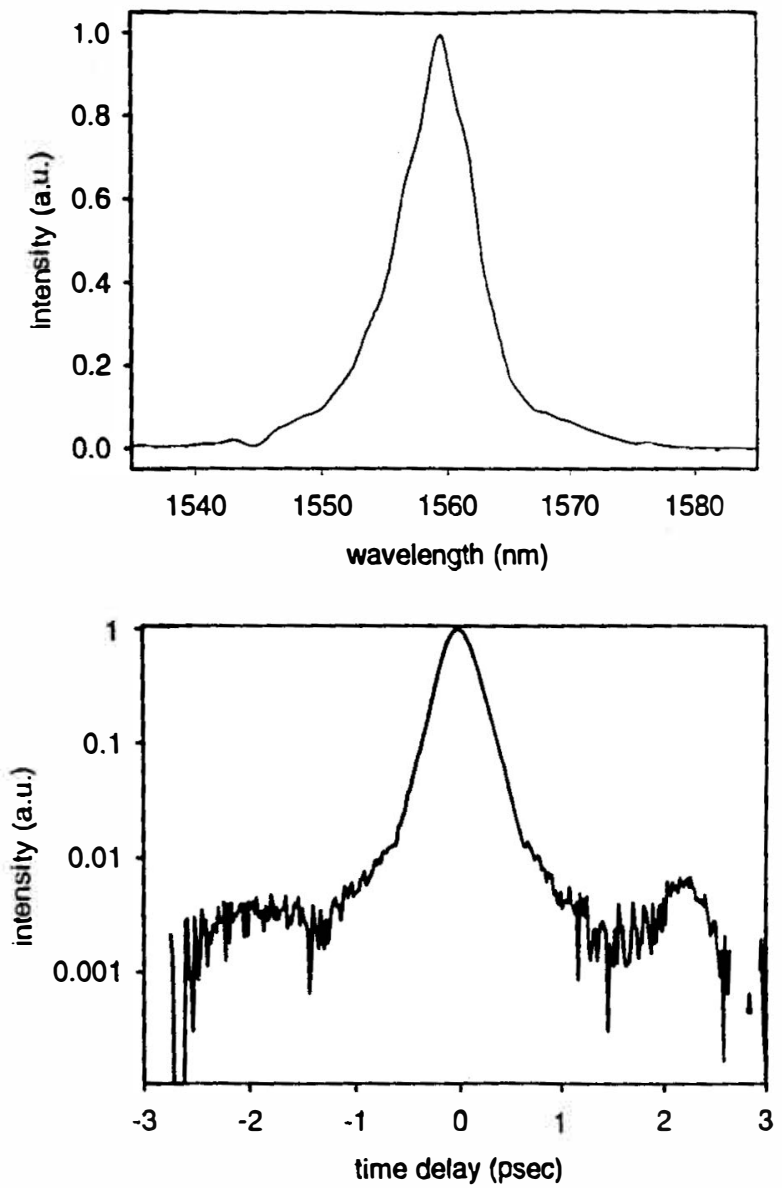

Fig. 3. Spectrum and autocorrelation of $12 \mathrm{~kW}$ peak power, near band width-limited 340 fs pulses amplified in a $30-\mu \mathrm{m}$-core diameter $\mathrm{Er}_{\mathbf{r}} / \mathrm{\zeta}$ tiber.

1. M.H. Muendel et al., in Conference on Lasers and Electro-Optics, 11 of 1997 OSA Technical Digest Series ( Optical Society of America Washington, D.C., 1997), paper CPD30. 